\title{
Effect of combined physical exercise program on improving the functional capacity and the cardiac autonomic modulation of Chagas cardiomyopathy
}

\author{
Diogo Van Bavel ${ }^{1,2}$ (D), Wallace Machado Magalhães de Souza ${ }^{1,2}$ (D), Yan de Britto Nery ${ }^{1}$ (D), \\ Juliana Nascimento Amorin $^{1}$ (D), Roberto Coury Pedrosa ${ }^{2}$ (D), Michel Silva Reis ${ }^{1,2,3}$ \\ ${ }^{1}$ Universidade Federal do Rio de Janeiro, Faculdade de Fisioterapia, Grupo de Pesquisa em \\ Avaliação e Reabilitação Cardiorrespiratória, Rio de Janeiro, RJ, Brasil; ${ }^{2}$ Universidade Federal \\ do Rio de Janeiro, Instituto do Coração Edson Saad, Rio de Janeiro, Faculdade de Medicina, RJ, \\ Brasil; ${ }^{3}$ Universidade Federal do Rio de Janeiro, Escola de Educação Física e Desportos, Rio de \\ Janeiro, RJ, Brasil.
}

Associate Editor: Maria Andréia Delbin/Unicamp, Campinas, SP, Brasil.

\begin{abstract}
Aims: Patients with Chagas cardiomyopathy $(\mathrm{ChC})$ could have a significant reduction in functional capacity (FC). This study aimed to report the effect of a 24-week combined physical exercise program on the FC of a patient with ChC. Methods: A woman, 44 years old, with positive serology for $\mathrm{ChC}$ in stage B2 has submitted the following assessments: i) Physical assessment: ii) Cardiopulmonary exercise test; iii) Test of 1-maximum Repetition, iv) Evaluation of cardiac autonomic modulation by heart rate variability (HRV). Results: The results obtained revealed a reduction in the anthropometric parameters and the SBP after training. Additionally, we observed an improvement in FC (aerobic and strength condiction) and cardiac autonomic modulation after 24 weeks of combined training. Conclusion: Our findings show that of a 24 -week combined physical exercise program improved either FC or HRVof the patient with $\mathrm{ChC}$.
\end{abstract}

Keywords: Chagas cardiomyopathy, aerobic training, strength training, functional capacity, heart rate variability.

\section{Introduction}

Patients with Chagas cardiomyopathy (ChC) with left ventricular dysfunction (LVD) could have impaired functional capacity. Cardiac remodeling induced by pathogens in $\mathrm{ChC}$ can lead to a progressive reduction in cardiac performance with an impact on the functional capacity of individuals. In this context, cardiac damage, abnormalities of the sympathetic-vagal balance, poor diet, chronic and systematic inflammatory process, medications, and physical inactivity could explain intolerance to physical exercise, muscle weakness, and worsening of the quality of life of patients with $\mathrm{ChC}^{1-3}$.

Oxygen consumption at peak effort $\left(\mathrm{VO}_{2}\right.$ peak), metabolic thresholds, ventilatory efficiency (VE/VCO2slope) with gravity marker, and presence of periodic breathing (PB) have been evaluated in the $\mathrm{ChC}^{1,2,4}$. However, few studies have investigated the effects of a combined formal exercise program with aerobic exercise and strength exercise in patients with $\mathrm{ChC}$ with LVD. This makes the case report potentially interesting when sharing the experience of our group in non-drug therapy in $\mathrm{ChC}$. In this context, the present case study aimed to report the effect of a 24-week combined physical exercise program on the clinical profile and $\mathrm{FC}$ of a patient with $\mathrm{ChC}$ with LVD without symptoms of heart failure.

\section{Case report}

A woman, 44-years-old, housewife, with positive serology for ChC, diagnosed 15 years ago, in stage B2 of the disease (altered electrocardiographic signal and left ventricular ejection fraction of $42 \%$ without HF clinic). The patient was using optimized and unchanged medication over the 24 weeks of the study (Carvedilol $6.25 \mathrm{mg}$ / day), had normal pulmonary function values with a forced vital capacity $(\mathrm{FVC})$ of $2.11 \mathrm{~L}$, and a forced expiratory volume in the first second $\left(\mathrm{FEV}_{1}\right)$ of $2.09 \mathrm{~L}$. Additionally, the patient had BMI characterizing grade I obesity, was sedentary, free of comorbidities such as hypertension, diabetes, dyslipidemia, or the coexistence of other diseases. Anthropometric, clinical, strength, and hemodynamic characteristics were presented in Table 1. This study was approved by the Ethics Committee of Hospital Universitario Clementino Fraga Filho da Universidade Fed- 
Table 1 - Anthropometric, clinical, strength, and hemodynamic values from a woman with Chagas Cardiopathy measured at baseline (T1), three (T3), and six (T6) months after 24-week combined physical training.

\begin{tabular}{|c|c|c|c|}
\hline Characteristics & T1 & T3 & T6 \\
\hline \multicolumn{4}{|l|}{ Anthropometric } \\
\hline Body mass (kg) & 85.7 & $84.4(-1.51 \%)$ & $83.4(-2.68 \%)$ \\
\hline BMI $\left(\mathrm{kg} / \mathrm{m}^{2}\right)$ & 33.5 & $33(-1.49 \%)$ & $32.6(-2.68 \%)$ \\
\hline SBP (mmHg) & 150 & $100(-33 \%)$ & $120(-20 \%)$ \\
\hline $\mathrm{DBP}(\mathrm{mmHg})$ & 90 & $70(-22 \%)$ & $80(-11 \%)$ \\
\hline MBP (mmHg) & 110 & $80(-27 \%)$ & $87(-21 \%)$ \\
\hline Percentage body fat (\%) & 37.5 & $35.7(-4.8 \%)$ & $33.1(-11.7 \%)$ \\
\hline WHR (cm) & 0.84 & $0.80(-4.7 \%)$ & $0.79(-5.9 \%)$ \\
\hline \multicolumn{4}{|l|}{ Cardiopulmonary Exercise Test } \\
\hline \multicolumn{4}{|l|}{ At rest } \\
\hline $\mathrm{VO}_{2}$ absolute $(\mathrm{L} / \mathrm{min})$ & 0.04 & $0.02(-50 \%)$ & $0.07(+75 \%)$ \\
\hline $\mathrm{VO}_{2}$ relative $(\mathrm{ml} / \mathrm{kg} / \mathrm{min})$ & 0.47 & $0.24(-51 \%)$ & $0.84(+79 \%)$ \\
\hline \multicolumn{4}{|l|}{ VAT } \\
\hline $\mathrm{VO}_{2}$ absolute $(\mathrm{L} / \mathrm{min})$ & 0.63 & $0.53(-15.8 \%)$ & $0.57(-9.5 \%)$ \\
\hline $\mathrm{VO}_{2}$ relative $(\mathrm{mL} / \mathrm{kg} / \mathrm{min})$ & 7.41 & $6.31(-14.8 \%)$ & $6.87(-7.2 \%)$ \\
\hline Time (s) & 146 & $324(121 \%)$ & $321(119 \%)$ \\
\hline Load (watts) & 40 & $65(62.5 \%)$ & $65(62.5 \%)$ \\
\hline \multicolumn{4}{|l|}{ Peak } \\
\hline $\mathrm{VO}_{2}$ absolute $(\mathrm{L} / \mathrm{min})$ & 1.2 & $0.85(-29 \%)$ & $0.89(-26 \%)$ \\
\hline $\mathrm{VO}_{2}$ relative $(\mathrm{mL} / \mathrm{kg} / \mathrm{min})$ & 13.7 & $10.12(-26.2 \%)$ & $10.72(-22 \%)$ \\
\hline Time (s) & 480 & $624(30 \%)$ & $612(27.5 \%)$ \\
\hline Load (watts) & 95 & $115(21 \%)$ & $115(21 \%)$ \\
\hline Ventilatory efficiency $\left(\mathrm{VE} / \mathrm{VCO}_{2}\right.$ Slope. $)$ & 26.25 & $21.99(-16.2 \%)$ & $18.27(-30.4 \%)$ \\
\hline \multicolumn{4}{|l|}{ 1-MR Test } \\
\hline Squat $(\mathrm{kg})$ & 62 & $76(22.5 \%)$ & $82(32.2 \%)$ \\
\hline Wide grip Lat pull down $(\mathrm{kg})$ & 45 & $48(6.6 \%)$ & $52(15.5 \%)$ \\
\hline
\end{tabular}

Legend: BMI: body mass index; DBP: diastolic blood pressure; MBP: mean blood pressure; SBP: systolic blood pressure; WHR: waist-to-hip ratio. VO ${ }_{2}$ : oxygen consumption; VE/VCO2 Slope: ventilatory equivalents of carbon dioxide; VAT: ventilatory anaerobic threshold; 1-MR: one maximum repetition. The values in parentheses represent percentage increases or decreases comparing T1 x T3 e T1 x T6.

eral do Rio de Janeiro- (CAAE No. 26421619.1.0000.5257).

The following assessments were carried out: i) Anamnesis and anthropometric assessment: measurements of body mass $(\mathrm{kg})$, height $(\mathrm{cm})$, body mass index (BMI), waist circumference $(\mathrm{cm})$, and fat percentage ${ }^{5}$; ii) Cardiopulmonary exercise test (CPET - VO2000 _ Portable Medical Graphics Corporation $\left.{ }^{\circledR}\right)$ : The test was performed on a cycle ergometer using a ramp protocol (incrementation of 10W/min) (Inbramed, Porto Alegre, Brazil). Oxygen consumption and workload at peak of CPET, ventilatory anaerobic threshold (VAT), ventilatory efficiency, and periodic breathing were obtained to assess $\mathrm{FC}^{6,7}$; iii) Test of 1-Maximum Repetition (1-MR): the maximum strength of
5 consecutive attempts of the squat and lat pulldown exercises was evaluated $^{8}$, and iv) Heart rate variability (HRV) was recorded by ECG system (Wincardio USB, Micromed, Brasília, Brazil) at rest in the supine and sitting positions. SDNN and SD2 indices (representative of the total HRV) and RMSSD, pNN50, NN50, and SD1 (representative of parasympathetic modulation) were analyzed ${ }^{9}$. In the sequence, the patient participated in a combined physical exercise program for 24 weeks, 3 times/week, and was accompanied by a multi-professional team. The routine was defined as (i) Aerobic training - 35 minutes on a cycle ergometer, with 5 minutes of warm-up at $40 \%$ of the VAT, 25 minutes at $100 \%$ of the VAT and 5 minutes of cool down; and, ii) Strength training - 5 exercises with 3 sets 
with repetitions varying according to periodization (60 $90 \% 1-\mathrm{MR}$ ) for the main muscle groups - squat, wide-grip pullup, back row, shoulder press, and sit-ups. Finally, the patient was evaluated at baseline (T1), in the third (T3), and sixth months (T6) after training. Table 1 shows the anthropometric and systemic blood pressure (SBP), cardiorespiratory characteristics, and muscle function at three moments. The results obtained revealed a reduction in the anthropometric parameters and the SBP after training. Regarding $\mathrm{VO}_{2}$ peak and VAT, an improvement was observed in workloads of CPET after six months of intervention. At moments $\mathrm{T} 3$ and $\mathrm{T} 6$, the VAT and $\mathrm{VO}_{2}$ peak occurred later than the baseline condition, revealing an improvement in FC. An increase in muscle strength in T3 was found in the squat and the wide-grip pull-up exercises. Figure 1 shows the ventilation in the three moments, where lower periodic breathing in $\mathrm{T} 3$ and $\mathrm{T} 6$ can be observed concerning the baseline condition, as well as a reduction in the $\mathrm{VE} / \mathrm{VCO}_{2 \text { slope }}$ of T1vs.T3 and T1vs.T6. It is possible to highlight the SDNN and RMSSD parameters
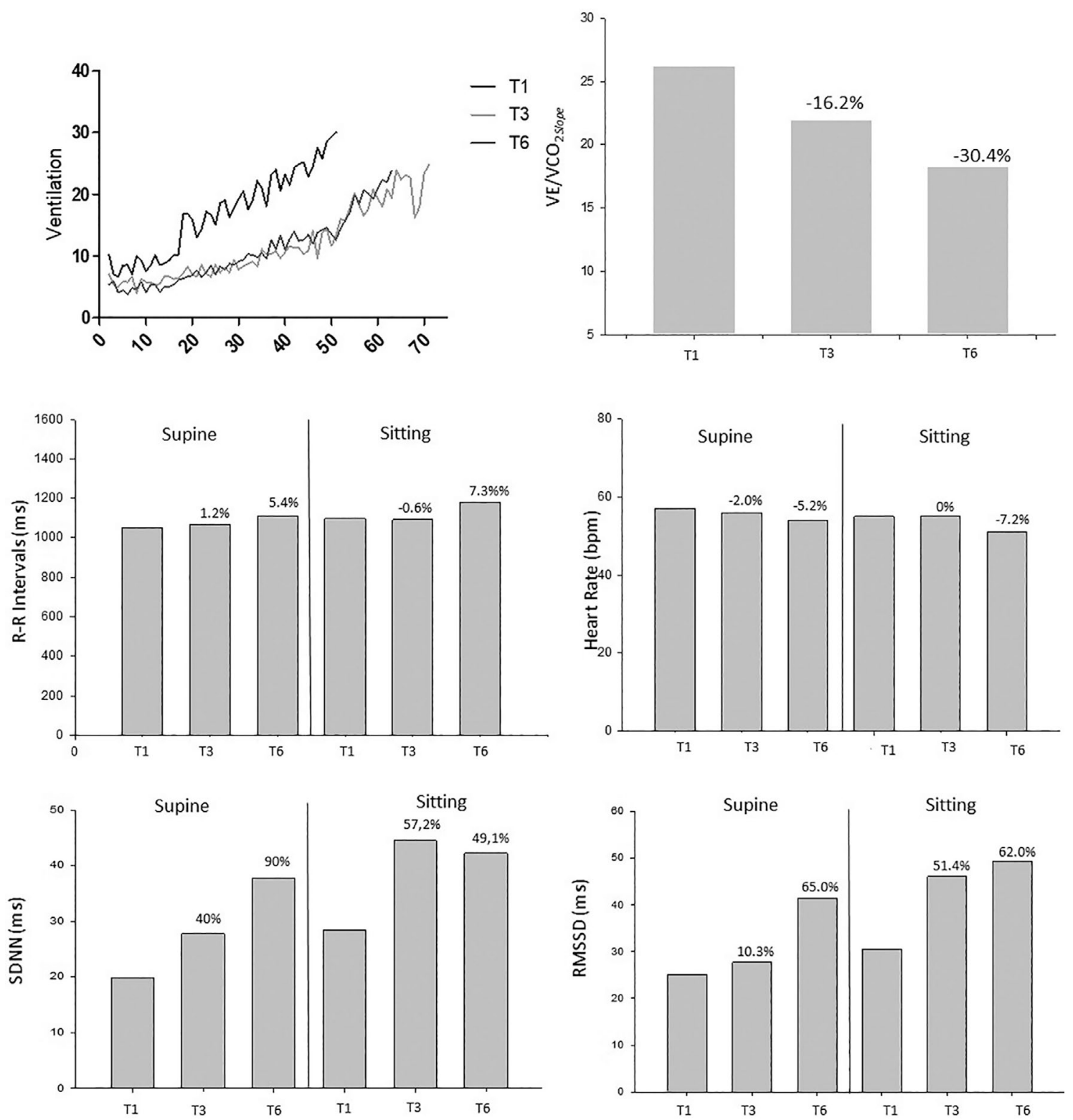

Figure 1 - Data on ventilation, ventilation efficiency, and heart rate variability from a woman from a woman with Chagas Cardiopathy throughout the intervention process in the lying and sitting position at baseline (T1), three (T3), and six (T6) months after 24-week combined physical training Legend: IRR: RR intervals; SDNN: Standard deviation of all normal RR intervals; RMSSD: Square root of the mean of the square of the differences between the adjacent normal RR intervals; NN50: Represents the adjacent RR intervals with a difference in duration greater than the 50s; pNN50: Percentage of adjacent RR intervals with a difference in duration greater than 50s; SD1: Scattering of points perpendicular to the identity line; SD2: Scattering of points along the identity line. 

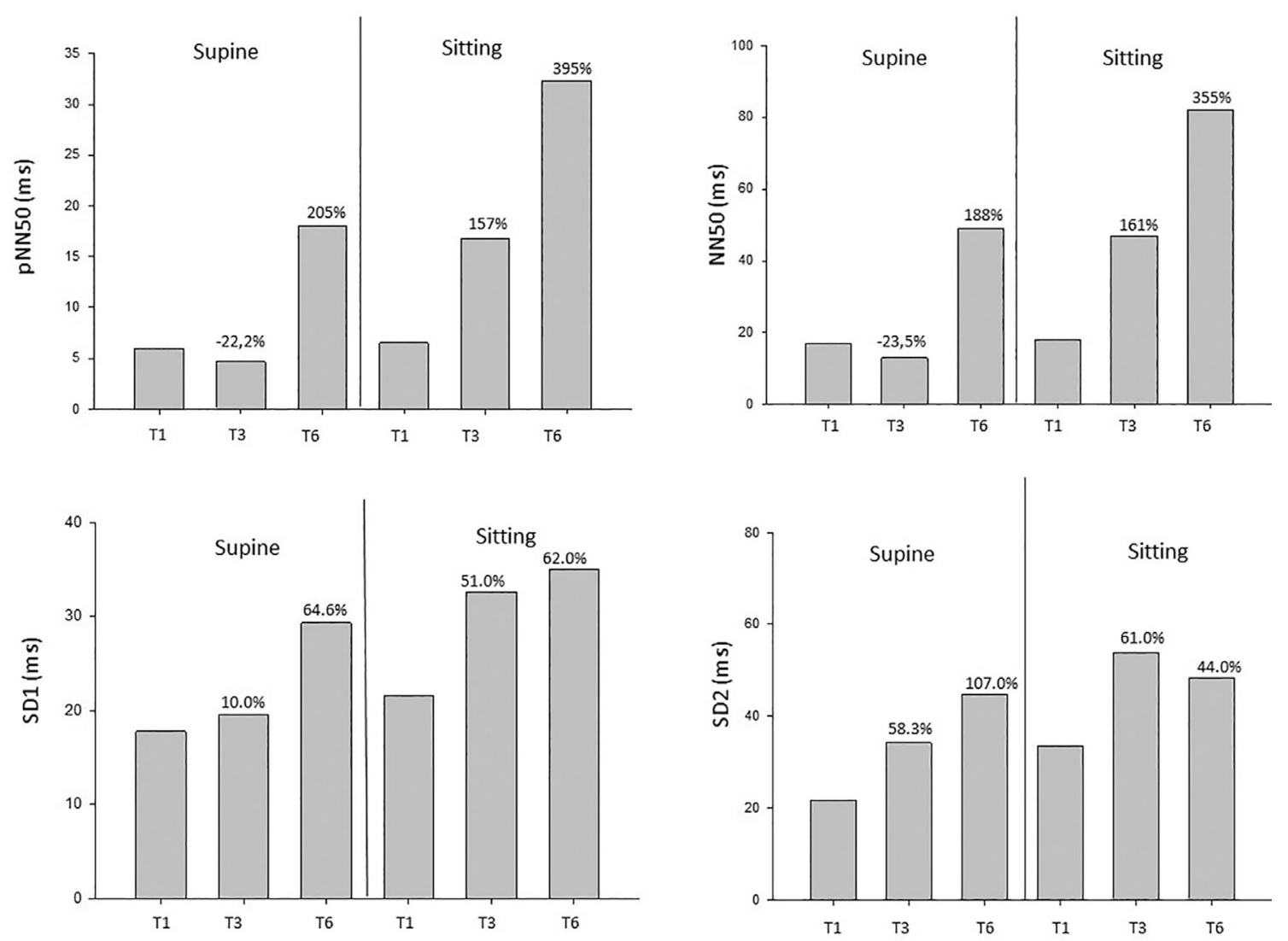

Figure 1 (cont.) - Data on ventilation, ventilation efficiency, and heart rate variability from a woman from a woman with Chagas Cardiopathy throughout the intervention process in the lying and sitting position at baseline (T1), three (T3), and six (T6) months after 24-week combined physical training Legend: IRR: RR intervals; SDNN: Standard deviation of all normal RR intervals; RMSSD: Square root of the mean of the square of the differences between the adjacent normal RR intervals; NN50: Represents the adjacent RR intervals with a difference in duration greater than the 50s; pNN50: Percentage of adjacent RR intervals with a difference in duration greater than 50s; SD1: Scattering of points perpendicular to the identity line; SD2: Scattering of points along the identity line.

in which percentage increases were observed in $\mathrm{T} 3$ and $\mathrm{T} 6$ and especially in the parameters related to vagal modulation in the sitting position.

\section{Discussion}

In the present case report, the patient showed an improvement in exercise capacity, an increase in peripheral muscle strength, and interestingly, lower periodic breathing with a reduction in $\mathrm{VE} / \mathrm{VCO}_{2 \text { slope }}$ after 3 and 6 months of combined physical training. Besides, it was possible to observe an improvement in anthropometric parameters and better cardiac autonomic modulation with reduced pressure values without changing the medication approach.

Patients with heart failure (HF) of chagasic etiology (the patient has no HF clinic) may have peripheral muscle dysfunction caused by a reduction in the volume of muscle mitochondria, in addition to a worsening in the action of oxidative enzymes, such as, for example, cytochrome $\mathrm{C}$ oxidase $^{10}$. This interferes with the use of oxygen, increas- ing the proportion of fibers with type II characteristics that determine early fatigue and decreased functional capacity and autonomy for activities of daily living ${ }^{10}$. However, the chronic stimulus of aerobic training combined with strength training can induce improvement of the left ventricular preload, with greater cardiac output and, mainly, adaptations of the trained peripheral muscles, potentiating the use of oxygen ${ }^{2}$. In this sense, the improvement in the physical exercise capacity in the T3 and T6 conditions can be attributed to the adaptations induced by the regular stimulus of the combined training in the improvement of the oxidative capacity. This finding should be highlighted, although the relative $\mathrm{VO}_{2}$ is lower in T6. There is solid evidence that peak $\mathrm{VO}_{2}$ increases with combined physical training. However, there is also the understanding that it is not an isolated parameter of improvement in patients with chronic cardiorespiratory diseases, mainly because cardiopulmonary testing is voluntary. In this context, a set of parameters (time of exhaustion, load, $\mathrm{VE} / \mathrm{VCO}_{2 \text { slope }}, \mathrm{VO}_{2}$ ) must be considered for the most assertive determination of improvement in functional capacity through combined 
training ${ }^{7}$. Additionally, despite the ventricular dysfunction presented by the patient, the improvement in $\mathrm{PB}, \mathrm{VE} /$ $\mathrm{VCO}_{2 \text { slope, }}$ and cardiac autonomic modulation may be related to greater cardiac performance ${ }^{9}$.

Finally, this case allows us to conclude that the of a 24-week combined physical exercise program improved the FC, muscle strength, and cardiac autonomic modulation of the patient with ChC. Besides, it modified the response of the $\mathrm{PR}$ and $\mathrm{VE} / \mathrm{VCO}_{2 \text { slope, }}$, suggesting less pulmonary congestion and better cardiac performance.

\section{Acknowledgments}

The authors would like to thank the National Council for Scientific and Technological Development (CNPq) and the Carlos Chagas Foundation for supporting Scientific Research in the State of Rio de Janeiro (FAPERJ) for financing the tools for the development of Research Group in Cardiorespiratory Evaluated and Rehabilitation (GECARE).

\section{References}

1. Costa HS, Lima MMO, Figueiredo PHS, Ávila MR, Menezes KKP, Mendonça VA, et al. Exercise tests in Chagas cardiomyopathy: an overview of functional evaluation, prognostic significance, and current challenges. Rev Soc Bras Med Trop. 2020;53:e20200100.

2. Bocchi EA. Exercise training in Chagas' cardiomyopathy: trials are welcome for this neglected heart disease. Eur J Heart Fail. 2010;12 (8):782-4.

3. Oliveira FP, Pedrosa RC. Ventilatory response during exercise among chronic Chagas cardiopathy patients. Sao Paulo Med J. 2006;124 (5):280-4.

4. Guazzi M, Raimondo R, Vicenzi M, Arena R, Proserpio C, Sarzi Braga S, et al. Exercise oscillatory ventilation may predict sudden cardiac death in heart failure patients. J Am Coll Cardiol. 2007;50 (4):299-308.

5. Sociedade Brasileira de Hipertensão, et al. I Brazilian guidelines on diagnosis and treatment of metabolic syndrome. Arq Bras Cardiol. 2005;84:1.

6. Floriano RS, Orsini M, Reis MS. Importance of cardiopulmonary testing for cardiovascular physical therapy. Fisioter Bras. 2019;20 (4):578-91.
7. Reis HV, Sperandio PA, Correa CL, Guizilini S, Neder JA, Borghi-Silva A, et al. Association of Oscillatory Ventilation during Cardiopulmonary Test to Clinical and Functional Variables of Chronic Heart Failure Patients. Braz J Cardiovasc Surg. 2018;33 (2):176-82.

8. Laoutaris ID, Adamopoulos S, Manginas A, Panagiotakos DB, Kallistratos MS, Doulaptsis C, et al. Benefits of combined aerobic / resistance / inspiratory training in patients with chronic heart failure. A complete exercise model? A prospective randomized study. Int J Cardiol. 2013;167 (5):1967-72.

9. Fenley A, Silva LC, Reis HV, Sampaio LM, Borghi-Silva A, Reis MS. Cardiorespiratory adjustments during the accentuation of respiratory sinus arrhythmia: influence from the time of maneuver on minute volume, a fraction of expired CO2, and heart rate variability. Fisioter Pesq. 2016;23 (1):68-73.

10. Silva RRD, Reis MS, Pereira BB, Nascimento EMD, Pedrosa RC. The additional value of anaerobic threshold in a general mortality prediction model in an urban patient cohort with Chagas cardiomyopathy. Rev Port Cardio. Circulation. 2017;36 (12):927-34.

\section{Corresponding author}

Michel Silva Reis, Ph.D. Research Group in Cardiorespiratory Evaluation and Rehabilitation (GECARE) / Department of Physical Therapy, Hospital Universitario Clementino Fraga Filho, Federal University of Rio de Janeiro. Prof. Rodolpho Paulo Rocco street, s/n, $2^{\circ}$ floor, Ilha do Fundão. 21941-913, Rio de Janeiro, RJ, Brazil. Telephone: +55 (21) 3938-9600.

E-mail: msreis@hucff.ufrj.br.

Manuscript received on September 30, 2020

Manuscript accepted on December 26, 2020

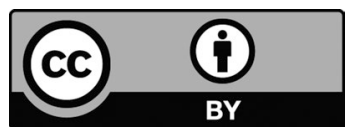

Motriz. The Journal of Physical Education. UNESP. Rio Claro, SP, Brazil - eISSN: 1980-6574 - under a license Creative Commons - Version 4.0 